ORIGINAL

\title{
Frecuencia de la infección por nemátodos gastrointestinales en ovinos y caprinos de cinco municipios de Antioquia
}

\section{Infection frequency of gastrointestinal nematode in goats and sheep of five municipalities in Antioquia}

\author{
Liseth Herrera O, ${ }^{1}$ Microb, Leonardo Ríos $0,{ }^{1}$ Ph.D, Richard Zapata S, ${ }^{1,2 *}$ M.Sc.
}

${ }^{1}$ Universidad de Antioquia, Escuela de Microbiología, Grupo de Investigación en Microbiología Veterinaria, Medellín, Colombia. ²Corporación Universitaria Lasallista, Facultad de Ciencias Administrativas y Agropecuarias, Caldas, Colombia. *Correspondencia: microbiolorich@gmail.com

Recibido: Marzo de 2012; Julio de 2013.

\section{RESUMEN}

Objetivo. Determinar la frecuencia de infección por nemátodos gastrointestinales en ovinos y caprinos de apriscos de algunos municipios de Antioquia. Materiales y métodos. Estudio descriptivo de corte realizado entre febrero y junio del 2011 en muestras de materia fecal de 84 caprinos y 11 ovinos distribuidos entre 2 y 132 meses de edad en 6 apriscos de Antioquia. Se determinó el recuento de huevos por gramo de heces (hpg) usando la técnica de Mcmaster, y para obtención del estadio larvario 3, la técnica de Corticelli y Lai. Para la identificación del género de las larvas se emplearon claves taxonómicas de nemátodos gastrointestinales de ovinos y caprinos. Se calcularon la frecuencia y asociación entre los datos epidemiológicos y la infección por tricostrongilidos mediante la prueba de independencia de Chi cuadrado, y prueba de contraste $U$ de Mann-Whitney. Resultados. La frecuencia de infección fue $86.6 \%$ y los nemátodos con mayor prevalencia fueron Haemonchus contortus (66.3\%), Oesophagostomum spp., (38.9\%), Trichostrongylus spp., (34.7\%) y Ostertagia spp., (24.2\%). Se encontró una diferencia estadísticamente significativa entre la proporción de infectados y no infectados según la raza. Conclusiones. Existen diferencias entre la frecuencia de infección de las razas Camuro, La mancha y Toggenburg, en comparación con otras razas de los mismos apriscos. Esta variación puede relacionarse con procesos de resistencia, reponiéndose a la infección, sobre los cuales se hace necesaria la realización de investigaciones enfocadas al estudio de mecanismos de acción en ovinos y caprinos de Antioquia.

Palabras clave: Cabras, epidemiología, infección, nematodos, ovinos, tracto gastrointestinal (Fuente: DECS). 


\section{ABSTRACT}

Objective. To determine the frequency of gastrointestinal nematode infection in goat and sheep folds of some municipalities of Antioquia. Materials and methods. A cross-sectional descriptive study was carried out between February and June 2011 in fecal samples of 84 goats and 11 sheep between 2 and 132 months of age in six folds in Antioquia. The number of eggs per gram of feces (epg) was determined using the McMaster technique, and the 3rd larval stage was obtained by means of Corticelli and Lai technique. For larval gender identification, gastrointestinal nematode taxonomical keys for goats and sheep were used. Tricostrongilides infection frequency and its association with epidemiological data was measured by means of chi-square test and comparison of proportions by column with $Z$ test and $U$ of Mann-Whitney contrast test. Results. Nematode infection frequency was $86.6 \%$ and Haemonchus contortus (66.3\%), Oesophagostomum spp (38.9\%), Trichostrongylus spp $(34.7 \%)$ and Ostertagia spp. (24.2\%) were the most prevalent nematodes. A statistically significant difference was found between infected and non-infected proportion per animal breed. Conclusions. There are differences between infection frequencies in Camuro, La mancha and Toggenburg breeds compared with other breeds in the same folds. These differences can be related with resistance and/ or recovering from infection, but more research focused in mechanisms of action in goats and sheep of Antioquia is necessary.

Key words: Epidemiology, gastrointestinal tract, goats, infection, nematoda, sheep (Source: DECS).

\section{INTRODUCCIÓN}

Los ovinos y caprinos son animales de producción múltiple siendo capaces de transformar forrajes de calidad baja en productos de gran valor, como son la lana, carne, leche y otros subproductos (1). En la explotación ovina y caprina se utilizan especies capaces de aprovechar los recursos naturales mediante el pastoreo (2). Sin embargo, diversas prácticas de manejo favorecen la infección por nemátodos gastrointestinales en ovinos y caprinos (3), lo que limita el desarrollo de la industria ovina y caprina (4), debido a bajas en la productividad (3).

La mayoría de los ovinos y caprinos son propensos a infecciones parasitarias comunes, debido a que su reproducción y cría se realiza de forma colectiva; siendo los nemátodos del grupo de los tricostrongilidos los parásitos más frecuentes en los apriscos (5). Estudios han evidenciado como un evento epizootiológico común las infecciones mixtas (6) con variedad de especies de nemátodos que se localizan en diferentes zonas del aparato digestivo, siendo las especies más comunes encontradas en el abomaso, Haemonchus contortus; en el intestino delgado, Bunostomun trigonocephalum, Cooperia curticel y Cooperia mcmasteri, Nematodirus filicollio, $N$. battus y $N$. spathinger, Trichostrongylus columbriformes, Trichostrongylus vitrinus y Strongyloides papillosus; y en el intestino grueso se ha reportado a Oesophagostomum venulosum, Trichuris ovis y Chabertia ovina (4).

La transmisión de los nemátodos intestinales ocurre en forma directa, a través de diferentes vías como ingestión, vía percutánea y lactogénica, las cuales se convierten en las principales formas de ingreso del parasito al hospedero; siendo los forrajes o aguas contaminadas con el estadio de larva 3 de estos parásitos las principales fuentes de contagio. Hembras y machos de los tricostrongilidos luego de alcanzar el estadio adulto en el aparato digestivo del rumiante, copulan con la posterior oviposición y liberación de huevos al medio a través de heces, estos se dispersan por las praderas y su eclosión y desarrollo de la larva hasta estadio 3 dependerá de condiciones de temperatura, $\mathrm{pH}$, humedad (7), y prácticas de manejo, las cuales pueden influir en la probabilidad de infección de los demás animales (8).

Según cifras de la FAO (2006) citadas por el Ministerio de Agricultura y Desarrollo Rural de Colombia, el inventario ovino-caprino mundial ubica a Colombia en el puesto 63 de los productores, después de países como China para el caso de la producción ovina; y Australia, Nueva Zelanda, India e Irán en referencia a la producción caprina; considerados estos como los mayores productores a nivel mundial (9). A nivel nacional la actividad productiva esta distribuida en todos los departamentos; sin embargo, se aprecia de forma más intensiva en los departamentos de Guajira, Magdalena, Atlántico, Bolívar, Sucre y Córdoba; asimismo Santander, Nariño, Putumayo, Cesar y el altiplano Cundiboyacense son también regiones en las que se registra una producción ovina y caprina importante (9). Colombia y particularmente Antioquia viene potenciando el desarrollo de la cadena ovino caprina a través de diferentes organizaciones gremiales que tienen como objetivo mejorar la productividad del sistema 
para lograr que el sector crezca (10), a pesar de esto, en esta región no se han realizado estudios epizootiológicos sobre nemátodos gastrointestinales en ovinos y caprinos, por tanto el objetivo de este estudio fue determinar la frecuencia de infección por nemátodos gastrointestinales en ovinos y caprinos de apriscos de algunos municipios de Antioquia en el año 2011.

\section{MATERIALES Y MÉTODOS}

Tipo de estudio. Se diseñó un estudio de tipo descriptivo con análisis de corte, con un muestreo no probabilístico, en ovinos y caprinos de 6 apriscos ubicados en la región norte y nororiente del departamento de Antioquia entre los meses de febrero y junio de 2011.

Área de estudio. Se realizaron muestreos en Ios apriscos: Las Carolinas (Guarne), Corcovado (La Ceja), Hogares Juveniles Campesinos (Bello), Universidad Nacional de Colombia Sede Medellín (Medellín), Cabriolas (Barbosa) y Potrerito (Bello). Las zonas de estudio presentan alturas que oscilan entre 1.300 y $2.150 \mathrm{msnm}$, con una temperatura que fluctúa en un rango de $16^{\circ} \mathrm{C}$ a $28^{\circ} \mathrm{C}$ y una precipitación media anual de 1656 $\mathrm{mm}$, con variaciones según la zona.

Población de estudio. La población de estudio estuvo conformada por un total de $95 \%$ semovientes: $84 \%$ ovinos de las razas Persa, Camuro, Katahdin/Camuro y Santa Inés, y $11 \%$ caprinos distribuidos en las razas Alpina, Saanen, Sannen/Alpina, Toggenburg, Anglonubiana y La Mancha. Se evaluaron pequeños rumiantes de ambos sexos y edades que oscilaron entre 2 y 132 meses, distribuidos en sistemas de confinamiento completo, semiconfinamiento $y$ pastoreo.

Recolección de muestras de materia fecal. Se recolectaron muestras de materia fecal directamente del recto de los animales, utilizando guantes de látex y se depositaron en frascos tapa rosca de $80 \mathrm{ml}$, parte de la muestra fue preservada con formalina al $10 \%(\mathrm{v} / \mathrm{v})$ para realizar el análisis cuantitativo de nemátodos y la otra parte se almacenó sin preservante para realizar el cultivo e identificación del estadio larvario 3. Cada muestra fue marcada con la identificación del animal, nombre del aprisco y fecha de recolección.

Toma de muestra de sangre y técnica del hematocrito. Se tomaron $4 \mathrm{ml}$ de sangre de la vena yugular de los ovinos y caprinos. La muestra fue recolectada en tubos vacutainer con EDTA y fue conservada a $4^{\circ} \mathrm{C}$ hasta su procesamiento. Posteriormente, se realizó la técnica de microhematocrito, la cual consistió en llenar un capilar con la muestra de sangre hasta $3 / 4$ partes, el cual luego fue centrifugado durante 5 minutos a 8000 rpm, y finalmente se evaluó en la tabla de lectura.

Cálculo de la carga parasitaria. Para el análisis cuantitativo de nemátodos en muestras de materia fecal fue empleada la técnica de recuento de McMaster (11). Para ello se pesaron $4 \mathrm{~g}$ de materia fecal y se mezclaron con $26 \mathrm{ml}$ de solución azucarada saturada de Sheather, luego el homogenizado fue filtrado a través de un tamiz metálico y posteriormente con el material filtrado se realizó el llenado de la cámara para la realización del recuento de huevos. El recuento de huevos morulados se hizo en la cámara triplechambered McMaster counting slide (Chalex corporation ${ }^{\circledR}$ ) utilizando para el cálculo de huevos por gramo (hpg) de materia fecal el factor de conversión 8 sugerido por la casa comercial.

Obtención del estadio larvario L3. Se utilizó la técnica de Corticelli y Lai para la obtención del estadio larvario L3 (12). De acuerdo con esta técnica se depositaron aproximadamente $10 \mathrm{~g}$ de materia fecal en una caja de Petri de $6 \mathrm{~cm}$ de diámetro, y está a la vez se colocó dentro de una caja de Petri de $9 \mathrm{~cm}$ de diámetro que contenía agua a una altura de $5 \mathrm{~mm}$ aproximadamente (cámara húmeda). El cultivo se incubó a temperatura ambiente durante 20 días. Transcurridos los 20 días se invirtió la caja de Petri pequeña con la muestra, dentro de la caja de Petri grande. Luego de 12 a 24 horas se tomó el agua con pipeta Pasteur y se depositó en un tubo de ensayo, se centrifugó a 4000 rpm por $4 \mathrm{~min}$, y se descartó el sobrenadante. El sedimento fue resuspendido en $50 \mu \mathrm{lde}$ formalina al $10 \%(\mathrm{v} / \mathrm{v})$ para preservar las larvas presentes hasta su caracterización.

Identificación del estadio larvario L3. La identificación del género del nematodo en el estadio larvario L3 se realizó mediante el uso de claves taxonómicas de nemátodos gastrointestinales de ovinos y caprinos. Estas claves se basan en el tamaño total del nematodo, el tamaño de la cola de la vaina, tamaño y forma del esófago, cantidad y forma de las células esticosomales y cuerpos refringentes (12).

Encuesta clínico-epidemiológica. Se tabuló la información en un instrumento tipo encuesta para la recolección de información de los animales y apriscos incluidos en el estudio. Estas variables fueron: sexo, edad, raza, semoviente, aprisco, tipo y frecuencia del antihelmíntico, tipo 
explotación y forraje principal, y valoración clínica mediante evaluación médico veterinaria.

Aspectos éticos. Se obtuvo la aprobación para la ejecución de la investigación, a través de un consentimiento informado leído y firmado por los representantes legales de los apriscos. Asimismo, en cumplimiento de las disposiciones expuestas en la resolución 008430 de 1993, el estudio fue evaluado y aprobado por el comité de ética en investigación animal de la Universidad de Antioquia en acta $\mathrm{N}^{\circ} 65$ del 29 de septiembre de 2010. También se consideraron las indicaciones descritas en la ley 84 del 27 de diciembre de 1989, para garantizar las buenas prácticas en los procedimientos a realizar en los ovinos y caprinos objeto de estudio y para garantizar su bienestar en todo el proceso.

Plan de análisis. Las características de los animales en el estudio se describieron mediante medidas de frecuencia y de resumen; la asociación entre las variables epidemiológicas (semoviente, raza, sexo, estado fisiológico, producción de leche, aprisco, sistema de explotación, rotación en potrero, forraje principal, tipo de bebedero y antihelmíntico) y la infección por tricostrongilidos se realizó mediante análisis bivariado con la prueba de independencia de Chi cuadrado y las comparaciones de la infección por tricostrongilidos según las variables edad, condición corporal, alzada, hematocrito, evaluación según escala famacha y frecuencia de antihelmíntico se realizaron con la prueba de contraste $U$ de Mann-Whitney. Un nivel de significancia de 0.05 fue utilizado en las pruebas. Los datos fueron analizados en el paquete IBM SPSS Statistics Software versión 19 del año 2011.

\section{RESULTADOS}

Este estudio incluyó 95 rumiantes pequeños, pertenecientes a caprinos $(88.4 \%)$ y ovinos $(11.6 \%)$, distribuidos entre los 2 y 132 meses de edad; la edad promedio fue 46 meses, el $50 \%$ de los individuos tenían 48 meses o menos y el $50 \%$ de los valores centrales de la edad estuvieron entre 12 y 72 meses. Estos rumiantes pertenecientes a 10 razas mostraron una mayor población de la raza Alpina (53.7\%), mayor frecuencia de hembras $(86.3 \%)$ y gran parte dedicada a la producción (36.8\%). Características de la población de estudio y los apriscos relacionados con la epizootiología de la nematodosis caprina se describen en las tablas 1 y 2 .

Se obtuvo una frecuencia de infección por nemátodos del $86.3 \%$. Siendo Haemonchus contortus (66.3\%), Oesophagostomum spp.
Tabla 1. Aspectos epizootiológicos de la población de estudio y los apriscos.

\begin{tabular}{llcc}
\hline & Variable & $\%$ & n \\
\hline Semoviente & Caprino & 88.4 & 84 \\
& Ovino & 11.6 & 11
\end{tabular}

Raza

\begin{tabular}{|c|c|c|c|}
\hline \\
\hline & Alpina & 53.7 & 51 \\
\hline & Anglonubiana & 8.4 & 8 \\
\hline & Camuro & 6.3 & 6 \\
\hline & Katadhin/camuro & 1.1 & 1 \\
\hline & La mancha & 3.2 & 3 \\
\hline & Persa & 3.2 & 3 \\
\hline & Saanen & 14.7 & 14 \\
\hline & Saanen/alpina & 1.1 & 1 \\
\hline & Santaines & 1.1 & 1 \\
\hline & Toggenburg & 7.4 & 7 \\
\hline \multicolumn{4}{|l|}{ Sexo } \\
\hline & Hembra & 86.3 & 82 \\
\hline & Macho & 13.7 & 13 \\
\hline \multicolumn{4}{|c|}{ Estado fisiológico } \\
\hline & Cría & 12.6 & 12 \\
\hline & Horra & 12.6 & 12 \\
\hline & Levante & 8.4 & 8 \\
\hline & Padrón & 4.2 & 4 \\
\hline & Preñada & 25.3 & 24 \\
\hline & Producción & 36.8 & 35 \\
\hline \multicolumn{4}{|c|}{ Aprisco } \\
\hline & Cabriola & 15.8 & 15 \\
\hline & Potrerito & 7.4 & 7 \\
\hline & Corcovado & 14.7 & 14 \\
\hline & Las Carolinas & 15.8 & 15 \\
\hline & UNAL† & 25.3 & 24 \\
\hline & $\mathrm{HJC} \neq$ & 25.1 & 20 \\
\hline \multicolumn{4}{|c|}{ Sistema de explotación } \\
\hline & Confinamiento completo & 51.6 & 49 \\
\hline & Pastoreo & 25.3 & 24 \\
\hline & Semiconfinamiento & 23.2 & 22 \\
\hline \multicolumn{4}{|c|}{ Rotación en potrero } \\
\hline & No & 52.6 & 50 \\
\hline & Sí & 47.4 & 45 \\
\hline \multicolumn{4}{|c|}{ Período de ocupación (días) } \\
\hline & 0 & 53.7 & 51 \\
\hline & 5 & 25.3 & 24 \\
\hline & 10 & 21.1 & 20 \\
\hline \multicolumn{4}{|c|}{ Forraje principal } \\
\hline & Estrella & 46.3 & 44 \\
\hline & Grama nativa & 7.4 & 7 \\
\hline & Maralfalfa & 31.6 & 30 \\
\hline & Pasto de corte & 14.7 & 14 \\
\hline \multicolumn{4}{|c|}{ Tipo de bebedero } \\
\hline & Cemento & 36.8 & 35 \\
\hline & Chupón & 14.7 & 14 \\
\hline & Plástico & 48.4 & 46 \\
\hline \multicolumn{4}{|c|}{ Antihelmíntico } \\
\hline & Doramectina & 21.1 & 20 \\
\hline & Febendazol & 7.4 & 7 \\
\hline & Febendazol/levamisol & 25.3 & 24 \\
\hline & Ivermectina & 30.5 & 29 \\
\hline & Levamisol & 15.8 & 15 \\
\hline \multicolumn{4}{|c|}{ Frecuencia de altihelmíntico (días) } \\
\hline & 30 & 25.3 & 24 \\
\hline & 45 & 15.8 & 15 \\
\hline & 90 & 14.7 & 14 \\
\hline & 120 & 15.8 & 15 \\
\hline & 180 & 7.4 & 7 \\
\hline & 280 & 21.1 & 20 \\
\hline
\end{tabular}

+ Universidad Nacional de Colombia Sede Medellín.

\# Hogares Juveniles Campesinos. 
Tabla 2. Aspectos epizootiológicos de la población de estudio.

\begin{tabular}{lccccc}
\hline & & CC & AL & VC & H \\
\hline \multirow{1}{*}{$\mathrm{N}$} & Válidos & 95 & 95 & 95 & 95 \\
Media & Perdidos & 0 & 0 & 0 & 0 \\
Mediana & & 3.0 & 71 & 2.6 & 30 \\
Moda & & 3.0 & 72 & 2.5 & 30 \\
Desv. típ. & & $3^{\mathrm{a}}$ & 72 & $2.0^{\mathrm{a}}$ & $27^{\mathrm{a}}$ \\
& & 0.7480 & 6.961 & 0.8091 & 4.631 \\
& Mínimo & 1.5 & 47 & 1.0 & 20 \\
Percentiles & Máximo & 5.0 & 88 & 4.5 & 45 \\
& 25 & 2.5 & 67 & 2.0 & 27 \\
& 50 & 3.0 & 72 & 2.5 & 30 \\
& 75 & 4.0 & 75 & 3.0 & 32 \\
\hline
\end{tabular}

CC: Condición corporal; AL: Alzada; VC: Valoración clínica (famacha); H: Hematocrito.

a. Existen varias modas. Se muestra el menor de los valores.

(38.9\%), Trichostrongylus spp. (34.7\%) y Ostertagia spp $(24.2 \%)$ los parásitos más frecuentes en los apriscos estudiados (Tabla 3).

Tabla 3. Frecuencia de positividad según clasificación taxonómica.

\begin{tabular}{lcc}
\multicolumn{1}{c}{ Nemátodo } & Negativo \% & Positivo \% \\
\hline Tricostrongilidos & 13.7 & 86.3 \\
Oesophagostomum spp & 61.1 & 38.9 \\
Haemonchus contortus & 33.7 & 66.3 \\
Trichostrongylus spp & 65.3 & 34.7 \\
Bunostomum spp & 90.5 & 9.5 \\
Ostertagia spp & 75.8 & 24.2 \\
Chabertia spp & 95.8 & 4.2 \\
Nematodirus spp & 97.9 & 2.1 \\
Cooperia spp & 89.5 & 10.5 \\
Strongyloides papillosus & 87.4 & 12.6 \\
Trichuris ovis & 97.9 & 2.1 \\
Skrjabinema ovis & 89.5 & 10.5
\end{tabular}

El $15.7 \%$ de los rumiantes presentaron cargas parasitarias de 700 o más huevos por gramo (hpg) de materia fecal, las cuales son consideradas como altas (Tabla 4).

Tabla 4. Distribución de la carga parasitaria según intensidad.

\begin{tabular}{cc}
\hline Carga parasitaria & \% de semovientes \\
\hline 200 o menos hpg MF* & 53.9 \\
201 -700 hpg MF & 30.4 \\
701 o más hpg MF & 15.7 \\
\hline
\end{tabular}

* Huevos por gramo de materia fecal

Al utilizar la prueba de contraste $U$ de Mann Whitney con un nivel de significancia de 0.05, no se encontró relación estadísticamente significativa entre la infección y las variables edad (p 0.071), condición corporal ( $p$ 0.257), alzada ( $p$ 0.231), evaluación según escala famacha ( $p$ $0.990)$, hematocrito ( $p$ 0.909) y frecuencia de tratamiento antihelmíntico ( $p$ 0.770). El análisis bivariado con la prueba de independencia Chi cuadrado mostró asociación estadísticamente significativa entre la infección y las variables raza ( $p 0.039)$, producción de leche ( $p$ 0.019), aprisco ( $p$ 0.001) y tipo de bebedero (0.001), (Tabla 5).
Tabla 5. Análisis bivariado entre variables fisiológicas, clínicas, productivas y de manejo animal con la positividad para nemátodos gastrointestinales.

\begin{tabular}{cccc}
\hline Variable & \% Neg & \% Pos & P \\
\hline $\begin{array}{c}\text { Semoviente } \\
\text { Caprino } \\
\text { Ovino }\end{array}$ & 11.9 & 88.1 & 0.163 \\
& 27.3 & 72.7 &
\end{tabular}

Raza

Alpina

Anglonubiana

Camuro

Katahdin/camuro

La mancha

Persa

Saanen

Saanen/alpina

Santaines

Toggenburg

Sexo

Hembra

Macho

Estado fisiológico

Cría

Horra

Levante

Padrón

Preñada

Producción

Producción de leche

No

Aprisco

Cabriola

Potrerito

Corcovado

Las Carolinas

UNAL

$\mathrm{HJC}$

Sistema de explotación

Pastoreo

Semiconfinamiento

Rotación en potrero

$$
\text { No }
$$

Sí

Período de ocupación (días)

0
5

5

Forraje principal

Estrella

Grama nativa

Maralfalfa

Pasto de corte

0.039

$5.9 \quad 94.1$

$\begin{array}{ll}25 & 75 \\ 50 & 50\end{array}$

$0 \quad 100$

$33.3 \quad 66.7$

$0 \quad 100$

7.192 .9

$0 \quad 100$

$42.9 \quad 57.1$

$14.3 \quad 85.7$

Tipo de bebedero

Cemento

Chupón

Plástico

Antihelmíntico

Doramectina

Febendazol

Febendazol/levamisol

Ivermectina

Levamisol

Frecuencia de antihelmíntico (días)

30
45

90

120

180

280
$11 \quad 89$

$30.8 \quad 69.2$

$8.3 \quad 91.7$

$8.3 \quad 91.7$

$37.5 \quad 62.5$

$0 \quad 100$

$8.3 \quad 91.7$

$17.1 \quad 82.9$

0.019

$20 \quad 80$

$2.9 \quad 97.1$

$0 \quad 100$

$0 \quad 100$

$46.7 \quad 53.3$

$4.2 \quad 95.8$

$20 \quad 80$

$16.3 \quad 83.7$

$4.2 \quad 95.8$

$18.2 \quad 81.8$

$16 \quad 84$

$11.1 \quad 88.9$

$15.7 \quad 84.3$

$4.2 \quad 95.8$

$20 \quad 80$

$11.4 \quad 88.6$

$28.6 \quad 71.4$

$23.3 \quad 76.7$

$0 \quad 100$

0.054

0.307

0.001

0.489

0.261

0.001

$31.4 \quad 68.6$

$0 \quad 100$

$4.3 \quad 95.7$

$20 \quad 80$

$14.3 \quad 85.7$

$4.2 \quad 95.8$

$24.1 \quad 75.9$

$0 \quad 100$

0.770 
Tabla 6. Análisis bivariado entre la positividad para nemátodos gastrointestinales y las variables relacionadas con el uso de antihelmínticos.

\begin{tabular}{|c|c|c|c|}
\hline & $\%$ Neg & $\%$ Pos & $\mathbf{p}$ \\
\hline \multicolumn{4}{|l|}{ Oesophagostomum spp. } \\
\hline \multicolumn{4}{|l|}{ Antihelmíntico } \\
\hline Doramectina (D) & 40 & 60 & 0.000 \\
\hline Febendazol (F) & 28.6 & 71.4 & \\
\hline Febendazol/levamisol (F/L) & 100 & 0 & \\
\hline Ivermectina (I) & 62.1 & 37.9 & \\
\hline Levamisol $(\mathrm{L})$ & 40 & 60 & \\
\hline \multicolumn{4}{|l|}{ Frec Antihelmíntico (días) } \\
\hline $30(F / L)$ & 100 & 0 & 0.000 \\
\hline $45(I)$ & 66.6 & 33.4 & \\
\hline $90(\mathrm{I})$ & 57.1 & 42.9 & \\
\hline $120(\mathrm{~L})$ & 40 & 60 & \\
\hline $180(F)$ & 28.6 & 71.4 & \\
\hline $280(D)$ & 40 & 60 & \\
\hline \multicolumn{4}{|l|}{ Haemonchus contortus } \\
\hline \multicolumn{4}{|l|}{ Antihelmíntico } \\
\hline Doramectina (D) & 25 & 75 & 0.011 \\
\hline Febendazol $(\mathrm{F})$ & 14.3 & 85.7 & \\
\hline Febendazol/levamisol (F/L) & 16.7 & 83.3 & \\
\hline Ivermectina (I) & 58.6 & 41.4 & \\
\hline Levamisol (L) & 33.4 & 66.6 & \\
\hline \multicolumn{4}{|l|}{ Frec Antihelmíntico (días) } \\
\hline $30(F / L)$ & 16.7 & 83.3 & 0.007 \\
\hline $45(\mathrm{I})$ & 73.3 & 26.6 & \\
\hline $90(\mathrm{I})$ & 42.8 & 57.2 & \\
\hline $120(\mathrm{~L})$ & 33.4 & 66.6 & \\
\hline $180(\mathrm{~F})$ & 14.3 & 85.7 & \\
\hline $280(D)$ & 20 & 80 & \\
\hline \multicolumn{4}{|l|}{ Trichostrongylus spp. } \\
\hline Antihelmíntico & 65 & 35 & 0.000 \\
\hline Doramectina (D) & 14.3 & 85.7 & \\
\hline Febendazol (F) & 25 & 75 & \\
\hline Febendazol/levamisol (F/L) & 100 & 0 & \\
\hline Ivermectina (I) & 60 & 40 & \\
\hline \multicolumn{4}{|l|}{ Levamisol (L) } \\
\hline Frec Antihelmíntico (días) & 25 & 75 & 0.000 \\
\hline $30(F / L)$ & 100 & 0 & \\
\hline $45(\mathrm{I})$ & 100 & 0 & \\
\hline $90(\mathrm{I})$ & 60 & 40 & \\
\hline $120(\mathrm{~L})$ & 71.4 & 28.6 & \\
\hline $180(\mathrm{~F})$ & 65 & 35 & \\
\hline \multicolumn{4}{|l|}{$280(D)$} \\
\hline \multicolumn{4}{|l|}{$\begin{array}{l}\text { Ostertagia spp. } \\
\text { Antihelmíntico }\end{array}$} \\
\hline Doramectina (D) & 70 & 30 & 0.028 \\
\hline Febendazol (F) & 57.1 & 42.9 & \\
\hline Febendazol/levamisol (F/L) & 62.5 & 37.5 & \\
\hline Ivermectina (I) & 96.6 & 3.4 & \\
\hline Levamisol (L) & 73.3 & 26.7 & \\
\hline \multicolumn{4}{|l|}{ Frec Antihelmíntico (días) } \\
\hline $30(F / L)$ & 62.5 & 37.5 & 0.050 \\
\hline $45(\mathrm{I})$ & 100 & 0 & \\
\hline $90(\mathrm{I})$ & 92.9 & 7.1 & \\
\hline $120(\mathrm{~L})$ & 73.3 & 26.7 & \\
\hline $180(\mathrm{~F})$ & 57.1 & 42.8 & \\
\hline $280(\mathrm{D})$ & 70 & 30 & \\
\hline \multicolumn{4}{|l|}{ Cooperia spp. } \\
\hline Doramectina (D) & 80 & 20 & 0.042 \\
\hline Febendazol (F) & 85.7 & 14.3 & \\
\hline Febendazol/levamisol (F/L) & 79.2 & 20.8 & \\
\hline Ivermectina (I) & 100 & 0 & \\
\hline Levamisol (L) & 100 & 0 & \\
\hline Frec Antihelmíntico (días) & & & \\
\hline $30(F / L)$ & 79.2 & 20.8 & 0.178 \\
\hline $45(\mathrm{I})$ & 100 & 0 & \\
\hline $90(\mathrm{I})$ & 100 & 0 & \\
\hline $120(\mathrm{~L})$ & 100 & 0 & \\
\hline $180(\mathrm{~F})$ & 85.7 & 14.3 & \\
\hline 280 (D) & 80 & 20 & \\
\hline
\end{tabular}

El análisis bivariado entre la infección por cada género o especie identificada arrojó que las variables tipo de antihelmíntico y la frecuencia de antihelmíntico se relacionan con la positividad para los parásitos Oesophagostomum spp. (p 0.000 y $\mathrm{p} 0.000)$, Haemonchus contortus ( $\mathrm{p} 0.011$ y $\mathrm{p} 0.007)$, Trichostrongylus spp. ( $\mathrm{p} 0.000$ y p 0.000), Ostertagia spp ( 0.028 y $p 0.050$ ) y Cooperia spp. (p 0.042), respectivamente (Tabla 6).

\section{DISCUSIÓN}

Los estudios realizados acerca de la epidemiología de los nemátodos gastrointestinales en ovinos y caprinos han sido reportados en España, y países de Latino América $(7,13)$. En España la prevalencia de nemátodos gastrointestinales en animales de pastoreo es de casi el $100 \%$ en algunas regiones. Según datos de diversos autores, la prevalencia en ovinos oscila entre el 68 y el $100 \%$ y en los bovinos entre el 41 y el $95 \%(7)$.

En este estudio se encontró una frecuencia de infección por nemátodos gastrointestinales del $86.3 \%$, y entre los agentes involucrados, Haemonchus contortus, Oesophagostomum spp., Trichostrongylus spp., y Ostertagia spp., fueron los nemátodos con mayor prevalencia en la población de estudio.

En Colombia no hay estudios de tricostrongilidos en pequeños rumiantes; sin embargo, los resultados obtenidos en esta investigación concuerdan con otros estudios realizados en bovinos (14) en los Llanos Orientales, donde se encontró Cooperia spp y Haemonchus spp como los endoparásitos dominantes, con incrementos poblacionales en las épocas de mayor precipitación (14). Resultados similares fueron reportados por Parra y Uribe (15) en la misma región; sin embargo, contrastan con los resultados obtenidos por Thullner (16) en el departamento de Córdoba, donde a pesar de encontrarse Cooperia spp., (C. punctata y C. oncophora), Haemonchus spp., (H. placei, $H$. similis) se obtuvo una alta frecuencia de Mecistocirrus sp (16).

A través del análisis bivariado con la prueba de independencia de Chi cuadrado se encontró una diferencia entre la proporción de infectados y no infectados según la raza $(p<0.039)$, siendo Camuro (50\%), La mancha $(66.7 \%)$ y Toggenburg $(57.1 \%)$ las razas que presentaron menor diferencia entre el porcentaje de rumiantes positivos y negativos, este hallazgo, según la literatura puede estar asociado con la variabilidad 
en la susceptibilidad de los animales a la infección según la raza. Algunas de las razas en las que se ha demostrado esta resistencia son: Blackbelly, St. Croix, Katahdin, Red maasai, Nali, Florida y sus cruces, Castellana, Gulf coast native, Scottish blackface, Crioula, Santa Ines y (17) Polaca de lana larga (18). Los pequeños rumiantes poseen mecanismos naturales de defensa contra los nemátodos gastrointestinales: la resistencia basada en estrategias inmunológicas que el animal posee en su código genético o que pueden adquirirse frente al reto parasitario para ejercer control sobre los nemátodos; y la capacidad de los animales de estar parasitados, recuperarse y/o adaptarse a la infección sin disminuir la productividad (19).

Para Haemonchus contortus no son bien conocidos los mecanismos de resistencia y capacidad de recuperación de ovinos; algunos autores sugieren que puede estar relacionada con factores inmunes, Alba-Hurtado y Muñoz-Guzmán, 2013 (20) plantean que a pesar de la producción de linfocitos CD4+ de forma similar entre razas de ovejas resistentes y susceptibles, estas últimas en comparación con las resistentes pueden producir relativamente más interferón gama (IFNy) y menos anticuerpos séricos específicos contra el parásito, menos eosinófilos sanguíneos y menos eosinófilos abomasales, indicando una relación entre la susceptibilidad a la infección por $H$. contortus y una respuesta inmune de tipo Th1 y una relación entre la resistencia a $H$. contortus y una respuesta inmune tipo Th2 (20). Asimismo, Gill y col en 2007 (21) encontraron relación entre la resistencia a $H$. contortus y los niveles de IgG1 e IgA sérica y como determinante genético la respuesta inmunológica mediada por anticuerpos tipo IgA fecales.

Por otro lado se encontró una asociación estadísticamente significativa $(p<0.019)$ entre el porcentaje de infección y la producción de leche, donde se encontró que el $97.1 \%$ de los animales que estaban en producción de leche fueron positivos para la infección por nemátodos gastrointestinales. Algunos estudios describen la existencia de una mayor susceptibilidad de las cabras lecheras de alta producción a las infecciones por nemátodos, asociado al período de gestación y lactancia, por la presentación de un estado de inmunosupresión, por lo cual se produce una mayor susceptibilidad del animal a la infección por helmintos, no se tiene claro el mecanismo por el cual ocurre esta alteración de la inmunidad; sin embargo, se ha relacionado con el efecto inmunosupresor de la prolactina y otros factores como la nutrición, el estado metabólico del animal y el medio ambiente (22).
Al evaluar la asociación entre la infección por tricostrongilidos y el aprisco se obtuvo un valor p 0.001 , en este hallazgo son notables las altas frecuencias de infección por nemátodos en cada aprisco, excepto en el aprisco Las Carolinas donde la distribución de individuos positivos (53.3\%) y negativos (46.7\%) fue semejante; lo que puede estar relacionado con el sistema de explotación por confinamiento completo que se practica en este aprisco (23) y con el uso de ivermectina con una periodicidad de 45 días, pues el uso de este antihelmíntico se relacionó de manera positiva en este estudio con la disminución en la proporción de animales positivos para tricostrongilidos.

Con respecto a las asociaciones entre la infección por nematodos y las variables descritas en el estudio, de acuerdo con los análisis realizados no se presenta confusión entre las variables evaluadas; en la tabulación epidemiológica realizada, las variables incluidas fueron las que según la literatura pueden asociarse a la infección; sin embargo, existen otros factores que no se han contemplado en este estudio.

Algunas investigaciones han demostrado que la ivermectina es el antihelmíntico con mayor eficacia contra las parasitosis causadas por nemátodos gastrointestinales en ovinos y caprinos, evaluada a través de la reducción de hpg de materia fecal (24). No obstante, su eficacia no alcanza el $100 \%$ y la resistencia antihelmíntica es un problema que en la industria ganadera ha venido en aumento; se ha reportado que la mayor prevalencia de la resistencia a los antihelmínticos en el mundo se presenta en parásitos de ovinos y caprinos (3). Dentro de los factores que se ha visto que influyen en la aparición de resistencia se contempla la frecuencia del tratamiento antihelmíntico y el número de tratamientos con la misma familia de antihelmínticos por tiempos muy prolongados (25). Según la literatura la resistencia de adultos de $H$. contortus en las ovejas se favorece por la capacidad de este nematodo para persistir por muchos meses en el rumiante, lo que le permite tanto adquirir resistencia como producir la próxima generación con esta característica de adaptación al hospedero (26).

La especie Haemonchus contortus es el parásito que independiente del antihelmíntico utilizado se encuentra infectando con una mayor proporción a ovinos y caprinos en este estudio. Este hallazgo puede estar relacionado con la posible adquisición de resistencia, ya reportada en diferentes estudios $(25,27)$; evento de adaptación del parásito para el cual se conocen como mecanismos la capacidad para realizar hipobiosis (28), adaptación a bajas concentraciones del químico y su exposición a períodos largos de tiempo (26). 
Este estudio encontró una asociación estadísticamente significativa entre la variable infección por género u especie de los tricostrongilidos identificados y las variables tipo de antihelmíntico y frecuencia de uso del antihelmíntico (Tabla 6). Se obtuvo una asociación estadísticamente significativa entre las variables Haemonchus contortus - antihelmíntico $(p<0.011)$, donde al observar los porcentajes de positividad entre los apriscos enzoóticos para esta especie de parásito $(100 \%)$ se evidencia la alta proporción de animales positivos independiente del antihelmíntico utilizado; sin embargo, en los apriscos donde utilizan ivermectina (cada 45 y 90 días) la frecuencia de animales negativos fue mayor (58.6\%). Un comportamiento similar presentaron las variables Ostertagia - antihelmíntico $(p<0.0028)$, donde se evidencia entre los apriscos protección parcial independiente del producto químico, con una mayor frecuencia de animales positivos (42.9\%) cuando se hace el tratamiento con febendazol cada 180 días.

Otro hallazgo fue la asociación Oesophagostomum - antihelmíntico $(p<0.000)$, donde el tratamiento implementado en el aprisco con febendazol/ levamisol podría estar relacionado con la ausencia del parásito (100\% negativos), similar comportamiento se obtuvo entre las variables Trichostrongylus spp. - antihelmíntico $(p<0.000)$ y entre Cooperia spp. - antihelmíntico ( $p<0.042)$, ya que en el primer caso el tratamiento implementado en dos apriscos con ivermectina está relacionada con la ausencia de los parásitos y el tratamiento con levamisol en otro aprisco podría estar relacionado con la ausencia del parásito (100\% negativos). En otros apriscos en los cuales el tratamiento se hace con otros químicos la eliminación no es completa, lo que concuerda con estudios donde se presenta a la ivermectina como uno de los antihelmínticos más efectivos (29). Estos hallazgos no son concluyentes; sin embargo, algunos estudios han evidenciado que la ausencia de parásitos en materia fecal de animales infectados puede darse debido a la hipobiosis que realizan Haemonchus contortus, Trichostrongylus, Teladorsagia (Ostertagia) circumcincta (30).

El estado hipobiótico de las larvas en la mucosa hace que estos estadios de desarrollo sean expuestos a concentraciones muy bajas del antiparasitario y que se genere un aumento en la población de refugio; es decir una población de larvas que no esta sujeta a la selección por los tratamientos químicos. Este es uno de los factores más importantes en la resistencia antihelmíntica, además, se favorece la adaptación de los nemátodos al químico (28). En $H$. contortus la resistencia por este mecanismo es muy común cuando se utilizan antihelmínticos que actúan contra larvas hipobióticas como los benzimidazoles, levamizol/pirantel y lactonas macrociclicas. Asimismo es el caso con el uso de moxidectina en comparación con el de ivermectina debido a que este último no posee una acción tan eficaz sobre las larvas hipobióticas (28).

Se concluye que los apriscos de ovinos y caprinos estudiados presentan una alta frecuencia de infección por nemátodos gastrointestinales, donde los géneros y/o especies más frecuentes son los nemátodos que en estadio L3 y L4 poseen la capacidad de realizar hipobiosis. Según la frecuencia de infección en las razas Camuro, La mancha y Toggenburg en comparación con otras razas de los mismos apriscos se puede concluir que existen diferencias no relacionadas con el azar, y que pueden estar relacionadas con procesos de resistencia y/o reponerse a la infección que se estén dando en los apriscos. Sin embargo, es necesario el desarrollo de líneas de trabajo enfocadas al estudio de los mecanismos de resistencia y recuperación frente a la infección por nemátodos gastrointestinales en las razas de ovinos y caprinos de los sistemas productivos de Antioquia y Colombia. Asimismo, se requiere estudiar las estrategias de adaptación de los nemátodos a los medicamentos utilizados para el control; y el desarrollo de metodologías de control alternativo y sostenible que no genere efectos nocivos sobre el sistema productivo, el medio ambiente y el consumidor.

\section{Agradecimientos}

Al Comité para el Desarrollo de la Investigación (CODI) por la financiación otorgada para el desarrollo de este estudio, y a los productores ovinos y caprinos del departamento de Antioquia que facilitaron sus apriscos para la realización de esta investigación. 


\section{REFERENCIAS}

1. Sievers G, Jara M, Cárdenas C, Núñez J. Estudio anual de la eliminación de huevos y ooquistes de parásitos gastrointestinales y larvas de nemátodos pulmonares en ovinos de una estancia en Magallanes, Chile. Arch Med Vet 2002; 34(1):37-47.

2. Ng'ang'a CJ, Maingi N, Kanyari PW, Munyua WK. Development, survival and availability of gastrointestinal nematodes of sheep on pastures in a semi-arid area of Kajiado District of Kenya. Vet Res Commun 2004; 28(6):491-501.

3. Márquez D. Resistencia a los antihelmínticos: origen, desarrollo y control. Rev Corpoica 2003; 4(1):55-71.

4. Vásquez Y, Morales G, Pino A, De Moreno L, De Combellas J. Cronobiología de la emisión de huevos de estróngilos digestivos en ovinos infectados en condiciones naturales. Zootec Trop 2001; 19(supl 1):279-287.

5. Bowman DD. Georgis' Parasitology for Veterinarians. 9th ed. Missouri: Saunders; 2008.

6. Morales G, Pino LA, Sandoval E, De Moreno L. Gastrointestinal nematode infection in ewes raised in an arid zone of Venezuela. Parasitol 2001; 25(1-2):36-39.

7. Cordero M, Rojo FA. Parasitología Veterinaria. Madrid: McGraw-Hill Interamericana; 1999.

8. Tariq KA, Chishti MZ, Ahmad F, Shawl AS. Epidemiology of gastrointestinal nematodes of sheep managed under traditional husbandry system in Kashmir valley. Vet Parasitol 2008; 158(1-2):138-143.

9. Ministerio de Agricultura y Desarrollo Rural. Observatorio Agrocadenas Colombia. La cadena ovinos y caprinos en Colombia. Bogotá: Ministerio de Agricultura y Desarrollo Rural; 2006.

10. Castellanos JG, Rodríguez JC, Toro WL, Luengas CL. Agenda prospectiva de investigación y desarrollo tecnológico para la cadena productiva cárnica ovinocaprina en Colombia. Bogotá: Ministerio de Agricultura y Desarrollo rural; 2010.
11. Shapiro LS. Pathology and Parasitology for Veterinary Technicians. 2nd ed. Delmar: Cengage Learning; 2010.

12. McMurtry LW, Donaghy MJ, Vlassoff $A$, Douch PGC. Distinguishing morphological features of the third larval stage of ovine Trichostrongylus spp. Vet Parasitol 2000; 90:73-81.

13. Torres-Acosta JF, Villarroel-Álvarez MS, Rodríguez-Arévalo $F$, Gutiérrez-Segura I, Alonso-Díaz MA. Diagnóstico de nemátodos gastrointestinales resistentes a bencimidazoles e imidazotiazoles en un rebaño caprino de Yucatán, México. Rev Biomed 2003; 14(2):75-81.

14. Rivera B, Parra D, García O, Aycardi E. Gastrointestinal parasites in calves in Colombia. Trop Anim Health Prod 1983; 15:107-114.

15. Parra D, Uribe LF. Epidemiología de nemátodos del bovino en el pie de monte de los Llanos Orientales de Colombia. Rev ACOVEZ 1990; 14(4):16-25.

16. Thullner $F$, Roqueme-Marrugo $L$, ParraGil D, Otte MJ. Investigaciones sobre la ocurrencia, epidemiología e importancia económica de los helmintos en terneros en el departamento de Córdoba, Colombia. Informe Técnico No. 10. Bogotá: Convenio Colombo-Alemán ICA-GTZ; 1991.

17. Amarante AFT, Amarante MRV. Breeding sheep for resistance to nematode infections. J Anim Vet Adv 2003; 2(3):147-161.

18. Bishop SC, Stear MJ. Modeling of host genetics and resistance to infectious diseases: understanding and controlling nematode infections. Vet Parasitol 2003; 115:147-166.

19. Aguilar-Caballero AJ, Torres-Acosta JFJ, Cámara-Sarmiento $R$, Hoste $H$, SandovalCastro CA. Inmunidad contra los nemátodos gastrointestinales: la historia caprina. Tropical and Subtropical Agroecosystems 2008; 9:73-82. 
20. Alba-Hurtado F, Muñoz-Guzmán MA. Immune Responses Associated with Resistance to Haemonchosis in Sheep. Biomed Res Int 2013. DOI: http://dx.doi. org/10.1155/2013/162158

21. Gill HS, Gray GD, Watson DL, Husband AJ. Isotype-specific antibody responses to Haemonchus contortus in genetically resistant sheep. Parasite Immunol 2007; 15:61-67.

22. Beasley AM, Kahn LP, Windon RG. The periparturient relaxation of immunity in Merino ewes infected con Trichostrongylus colubriformis: Endocrine and body compositional responses. Vet Parasitol 2010; 168:51-59.

23. Odoi A, Gathuma JM, Gachuiri $\mathrm{CH}$, Omore A. Risk factors of gastrointestinal nematode parasite infections in small ruminants kept in smallholder mixed farms in Kenya. BMC Vet Res 2007; 3:6

24. Bentounsi B, Attir B, Meradi S, Cabaret J. Repeated treatment faecal egg counts to identify gastrointestinal nematode resistance in a context of low-level infection of sheep on farms in eastern Algeria. Vet Parasitol 2007; 144:104-110.

25. Silvestre A, Leignel V, Berrag B, Gasnier N, Humbert JF, Chartier C, et al. Sheep and goat nematode resistance to anthelmintics: pro and cons among breeding management factors. Vet Res 2002; 33:465-480.
26. Coles GC. Cattle nematodes resistant to anthelmintics: why so few cases? Vet Res 2002; 33(5):481-489.

27. Baker KE, George SD, Stein PA, Seewald W, Rolfe PF, Hosking BC. Efficacy of monepantel and anthelmintic combinations against multiple-resistant Haemonchus contortus in sheep, including characterisation of the nematode isolate. Vet Parasitol 2012; 186:513-517.

28. Márquez D. Resistencia a los antihelmínticos en nemátodos de rumiantes y estrategias para su control. Bogotá: CorpoicaColciencias; 2007. [Fecha de acceso 1 de noviembre de 2011] URL disponible en: http://www.corpoica.org.co/SitioWeb/ Archivos/Publicaciones/Antihelmnticos.pdf

29. Muñoz JA, Angulo F, Ramírez R, Vale O, Chacín E, Simoes D, et al. Eficacia Antihelmintica de Doramectina $1 \%$, Ivermectina $1 \%$ y Ricobendazol $15 \%$ frente a nemátodos gastrointestinales en ovinos de pelo. Rev Científica FCV-LUZ 2008; 18(1):12-16.

30. Morgan ER, Van Dijk J. Climate and the epidemiology of gastrointestinal nematode infections of sheep in Europe. Vet Parasitol 2012; 189(1):8-14 DOI: dx.doi. org/10.1016/j.vetpar.2012.03.028 